\title{
COLLIDING WINDS IN O-TYPE BINARIES
}

\author{
DOUGLAS R. GIES \\ Department of Physics and Astronomy \\ Georgia State University, Atlanta, GA 30303-3083, U.S.A. \\ I: gies@chara.gsu.edu
}

\begin{abstract}
In close binary systems of O-type stars, the individual stellar winds will collide between the stars to form shock fronts. The existence of the shock region can be established through observations of excess X-ray emission and orbital phase-related variations in UV wind features and optical emission lines. Here I report on work in progress on the orbital variations of the UV wind lines in a large sample of O-binaries that have been observed with the International Ultraviolet Explorer satellite. In most of these binaries, the blue absorption trough of the P-Cygni lines weakens when the strong wind component is behind its companion, in accordance with expectations for colliding winds. I describe how the radial velocity variations of the wind features differ from the orbital motions. Finally I discuss observations of $\mathrm{H} \alpha$ emission in these systems, with emphasis on HD 47129 (Plaskett's star). Such optical emission lines act as a probe of high density regions in the winds.
\end{abstract}

Key words: stars: O-type stars - winds - close binaries

\section{Introduction}

The search for the effects of colliding winds in WR+O binaries is closely related to similar work on $\mathrm{O}+\mathrm{O}$ binary systems. The mass loss rates in $\mathrm{O}$-type stars $\left(-\dot{M} \sim 10^{-6}-10^{-5} \mathrm{M}_{\odot} \mathrm{yr}^{-1}\right)$ are smaller than in WR stars, but this mass flux is sufficient to create a high temperature and high density bow shock where the winds collide between the component $O$ stars. It is important to study wind outflows in $\mathrm{O}+\mathrm{O}$ binaries since these systems represent the precursors of the WR+O binaries, and, unlike the WR stars, they offer a "clear" view of the component photospheres (which better constrains the physical parameters of the stars).

The structure of the shocks formed in colliding wind binaries was studied through hydrodynamical simulations by Stevens, Blondin \& Pollock (1992), and their experiments show how a bow shock forms which wraps around the star with the weaker wind. The hot $\left(10^{7} \mathrm{~K}\right)$ gas associated with the shock will produce excess X-ray emission, and Chlebowski \& Garmany (1991) find evidence for this X-ray enhancement in Einstein observations of tight, detached $\mathrm{O}+\mathrm{O}$ binaries, whose components are of similar luminosity and are closer to each other than about five times the sum of their radii. In such binaries, the winds of both stars will be accelerated to high enough speeds and shock densities to produce a significant excess X-ray flux.

The presence of a bow-shock in the volume around a binary can cause large-scale changes in the velocity and density structure of the individual winds, and thus the shapes of the UV wind lines (formed by resonant scat- 
tering) may appear different with changes in orientation through the orbital cycle (Stevens 1993). Such arguments led to early studies of the UV wind lines in the O-type binaries 29 UW CMa (McCluskey \& Kondo 1976; Drechsel et al. 1981) and Plaskett's star = HD 47129 (Heap 1981; Sahade \& Brandi 1991).

Optical emission lines (such as $\mathrm{H} \alpha$ ) offer a complementary approach to the study of colliding winds in $\mathrm{O}$-type binaries. These emission lines are produced by recombination (proportional to density squared), and thus they act as a probe of regions of dense circumstellar gas. Much of the observational work on $\mathrm{H} \alpha$ emission in O-type binaries was done by Struve and collaborators some forty years ago (29 CMa: Struve et al. 1958; Plaskett's star: Struve, Sahade \& Huang 1958), long before there was an appreciation of the massive stellar winds of $\mathrm{O}$-type stars.

Here I describe the current picture of the orbital variations in the UV wind lines and optical emission lines, and how they can be interpreted in a colliding winds model.

\section{P-Cygni profiles}

Stevens (1993) has made Monte-Carlo simulations of photon scattering in colliding wind binaries to study the orbital variations in P-Cygni lines. He presents results for an idealized system of equal luminosity stars, with equal momentum in their winds, observed from the orbital plane $\left(i=90^{\circ}\right)$. The models do not include the orbital motions of the stars, but since orbital velocities are small compared to wind speeds, these models should offer broad guidance about what we might expect to observe. Three of these simulations are immediately relevant to $\mathrm{O}+\mathrm{O}$ binaries. (1) When both stars have optically thick winds, minimal variability is found since scatterers in the wind of the foreground star always compensate for lost scattering ions from the star behind that are blocked by the bow shock. (2) However, when only one wind is optically thick, the P-Cygni line displays large changes when the star with the optically thin wind is in front. The extreme blueedge velocity, $v_{\text {edge }}$, declines (since the wind of the star behind strikes the bow shock before reaching terminal velocity) and the absorption trough weakens (since photons from the foreground star stream unimpeded in the observer's direction). (3) If the winds have different terminal velocities, the model profiles show significant variations in $v_{\text {edge }}$ as the fast and slow winds pass in front of the system.

The realization that colliding winds can cause orbital variations in the UV wind lines provided the motivation to search for variations in a sample of $25 \mathrm{O}+\mathrm{O}$ close-binary systems that have been repeatedly observed in high dispersion mode (SWP/H) with the International Ultraviolet Explorer satellite (IUE). This group represents most of the double-lined systems in 
sky brighter than $V<8$. At the time of writing, our studies are complete for four binaries: AO Cas (O9III+O8V, Gies \& Wiggs 1991), Plaskett's star (O7.5I+O6I, Wiggs \& Gies 1992), 29 CMa (O8I+B0I, Wiggs \& Gies 1993), and $\iota$ Ori (O9III+B1III, Gies, Wiggs \& Bagnuolo 1993). We obtained new spectra for 8 systems, but for the rest, there are a sufficient number of archival spectra for an initial analysis (thanks in large measure to the heroic efforts of Sally Heap, Bob Koch, Dave Stickland, and other IUE observers). Here I present a summary of the preliminary results on the orbital variations in several key components of the P-Cygni features: the equivalent widths of the absorption trough and emission peak, and the radial velocities of the emission peak and blue-edge.

Absorption variations: The most compelling evidence for colliding winds is the widespread occurrence of a reduction in the absorption trough strength near inferior conjunction of the weak wind star ("secondary" in front; see LZ Cep in Fig. 1). For each system, I grouped the spectra in two bins according to placement relative to the line of the nodes ("primary in front" and "primary behind"), and then calculated the mean equivalent widths. The results are compared to the case of no variability (a line of unit slope) in Fig. 2. There is a consistent trend among most 0 -type binaries to show decreased absorption when the primary is "behind." This effect is similar in character to what Stevens (1993) found in models with winds of differing optical depth (case 2 above) which results from a reduction in scattering ions surrounding the weak wind secondary and dilution by photons from the secondary. The systems with nearly equally strong winds (those with similar spectral intensities, $\Delta m \approx 0$ ) tend to display little orbital variability (located along the diagonal line in Fig. 2) in agreement with Stevens' model for winds of equal optical depth (case 1 above). The few cases that lie above the diagonal line in Fig. 2 are systems where the so-called "secondary" probably has the stronger stellar wind.

The best example of a system dominated by the wind of the "secondary" is Plaskett's star (HD 47129). The nature of the secondary was revealed through an analysis of its UV photospheric spectrum by Bagnuolo, Gies \& Wiggs (1992) who applied the Doppler tomography algorithm to separate the individual spectral contributions of the two stars. The tomography algorithm uses an iterative least-squares scheme to reconstruct the two component spectra from an ensemble of composite spectra using information on the individual radial velocity curves and the magnitude difference. Analysis of the reconstructed spectra yields the spectral classification and projected rotational velocity of each star, and the spectra are potentially useful for abundance studies. The secondary in Plaskett's star is a rapid rotator $\left(v \sin i=310 \mathrm{~km} \mathrm{~s}^{-1}\right)$, and it has eluded easy detection in the past because its spectral lines are broad and shallow. The N IV $\lambda 1718$ feature appears as a P-Cygni profile in the secondary's reconstructed spectrum and as an ab- 


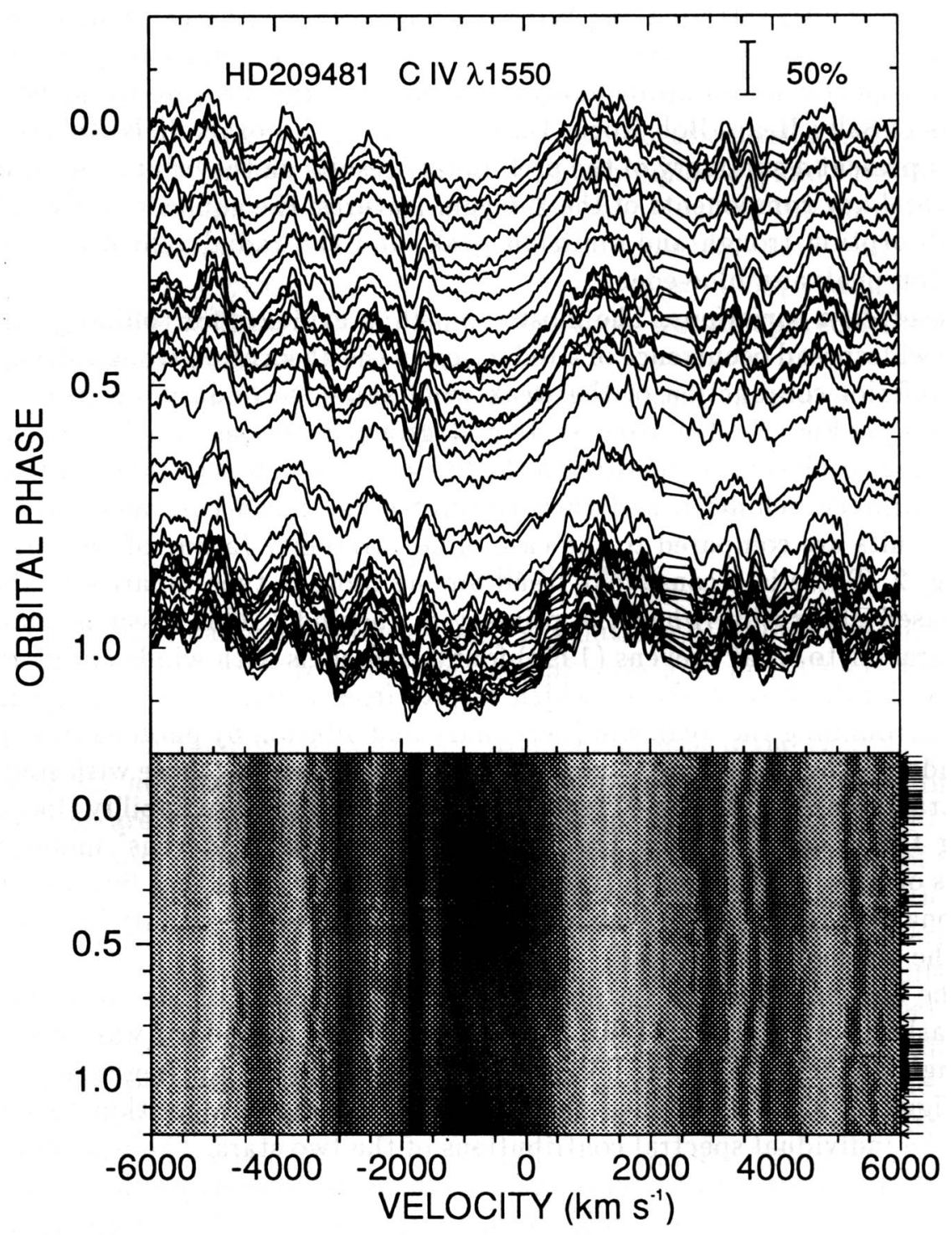

Fig. 1. CIV $\lambda 1550$ profiles of the binary LZ Cep plotted against radial velocity (based on the blue member of the doublet). The spectra in the upper panel are arranged with the continuum level (set to unity) located at the orbital phase of observation (from the orbit of Howarth et al. 1991). The lower gray-scale representation of the spectral variations is the result of a linear, orbital phase interpolation of the spectra. Note the decrease in absorption strength near superior conjunction of the primary (phase 0.8 ). 


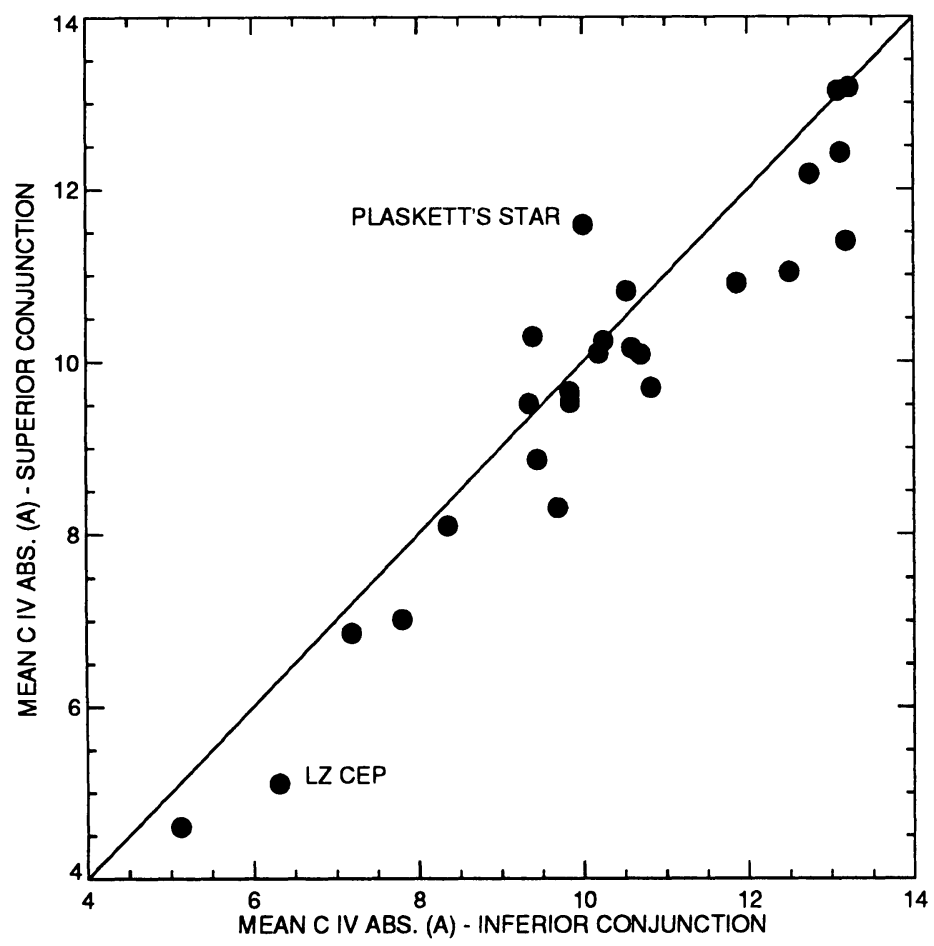

Fig. 2. A comparison of the mean $C_{I V} \lambda 1550$ absorption equivalent widths (in $\AA$ ) for spectra binned according to the primary's placement in front of $(x$-axis) or behind $(y$-axis) the secondary. Most systems display decreased absorption (below the diagonal line) when the primary is behind the secondary, as expected from colliding wind models.

sorption line in the primary's spectrum. This indicates that the secondary has the stronger wind, and thus the anomalous position of Plaskett's star in Fig. 2 results from misidentification of the dominant wind component.

Emission variations: The emission strength is generally constant except in eclipsing systems where the emission appears stronger at conjunctions. Most of this variation is due to a change in the ratio of emission intensity (approximately constant) to continuum flux (which decreases during eclipses). The amplitude of variation can then be calculated from the photometric light-curve, and the predicted conjunction brightenings usually are similar to those observed. An interesting exception is the Si IV $\lambda 1400$ wind feature in the spectrum of $29 \mathrm{CMa}$ in which the emission maxima at the conjunctions substantially exceed the predicted brightenings. One explanation could be the presence of a density enhancement (and an excess of scattering ions) along a planar bow-shock midway between the stars which would have its 
largest projected area on the sky at the conjunctions.

Peak-velocity-variations: The radial velocity of the red peak often shares the orbital motion of the strong wind ("primary") star, but the derived semiamplitude is usually smaller than that of the primary (e.g., AO Cas). In cases where the winds are of comparable strength (e.g., Plaskett's star), no variation is observed. The main scattering contributors to the emission peak are relatively close to the star, and thus this part of the profile should partially reflect the star's orbital motion. The smaller observed semi-amplitude probably results from line blending (emission components from both stars never fully resolved), and/or a focusing of the wind towards the secondary (when the primary is close to filling its Roche surface; Friend \& Castor 1982) which shifts the scattering distribution towards the center of mass.

Blue-edge velocity variations: The velocity of the blue absorption edge, $v_{\text {edge, }}$ generally shows no coherent phase-related variability. This lack of orbital motion is probably due to the great distance of the scattering ions from the star (near terminal velocity); here the integrated column density includes wind contributions that originated at all orbital phases, and any orbital variations are effectively averaged out. The absence of marked changes at the conjunctions is probably due to the characteristics of the binaries in our sample; most are double-lined systems with similar components and similar terminal velocities which would display little $v_{\text {edge }}$ variability (see model 1 of Stevens 1993, described above).

\section{Optical emission lines}

The optical emission lines offer an important complementary means to study the high density regions in the wind (close to shock fronts and photospheres). I have obtained high $\mathrm{S} / \mathrm{N}$ spectra of $\mathrm{H} \alpha$ in four binaries with the 2.1-m telescope and coudé spectrograph at the University of Texas McDonald Observatory, and these systems show a great diversity in properties. The velocity and intensity variations of $\mathrm{H} \alpha$ can be understood in terms of the colliding winds model, and the derived bow shock placement can range from close to secondary (AO Cas) to midway between the stars $(29 \mathrm{CMa})$ or nearby the primary (Plaskett's star).

The $\mathrm{H} \alpha$ emission in Plaskett's star has two components, a broad stationary feature which displays large night-to-night intensity variations (unrelated to orbital phase) and a sharp emission "spike" that follows the velocity curve of the primary but with a small phase lag (see Fig. 3). The radial velocity curve of the "spike" component can be described as the vector sum of orbital motion (orthogonal to the axis joining the stars) and gas flow (along the axis) The component due to orbital motion is directly related to the emission placement along the axis between the stars, and this vector decomposition leads to a source location coincident with the primary and 


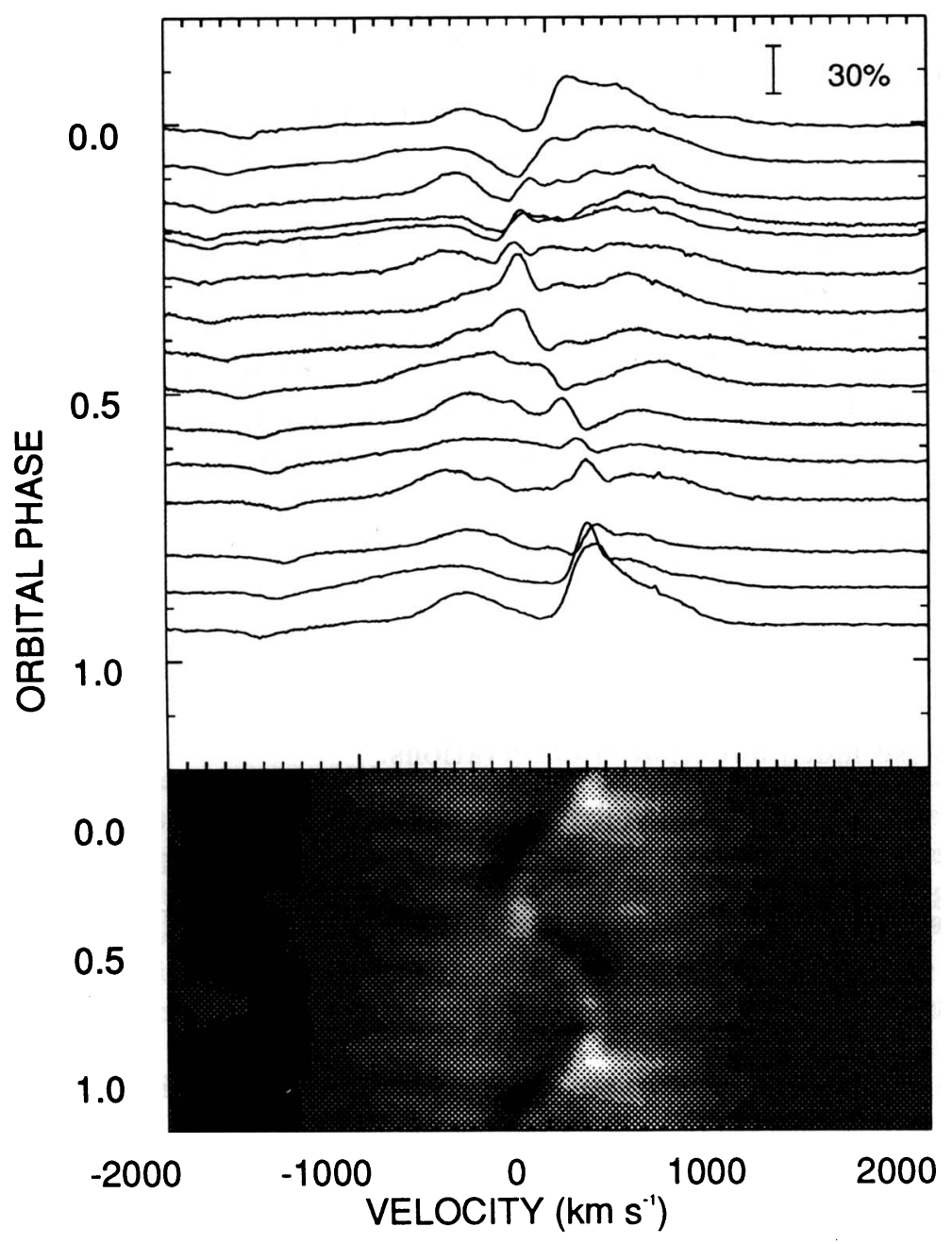

Fig. 3. H $\alpha$ emission in Plaskett's star (HD 47129) in the same format as Fig. 1. The faint He II $\lambda 6527$ line (at $v_{r}=-1624 \mathrm{~km} \mathrm{~s}^{-1}$ ) follows the velocity curve of the primary star.

to a gas flow directed from secondary to primary. This emission cloud probably represents post-shock gas in the vicinity of the primary and bounded by a bow shock in a wide cone around the primary. Both the UV wind and $\mathrm{H} \alpha$ profiles indicate that the axis of the shock suffers a large Coriolis deflection $\left(\sim 50^{\circ}\right)$. The high velocity $\mathrm{H} \alpha$ emission, on the other hand, shows no evidence of orbital motion, and it probably forms in high density knots in the shock region between the stars. The time-scale for variations in the high-velocity wings is comparable to the wind flow time between stars. 


\section{Summary}

The orbital variations of the UV wind lines act as a important probe of the large scale wind geometry in $\mathrm{O}+\mathrm{O}$ binary systems. A key observation is the widespread occurrence of absorption reductions when the strong wind star is behind; this indicates that colliding wind effects are present in most O-binaries. New but difficult line-transfer calculations are needed to fully exploit the observed variations, but such studies have great potential for unravelling the wind properties. A complementary task is the study of orbital variations in $\mathrm{H} \alpha$ and other emission features which contain important clues about the dense circumstellar gas regions. The combined data can be used to map out the location and velocity of gas outflows in these systems.

A census of the wind collision effects in a large sample of binaries will help elucidate the nature of colliding wind shocks, the rôle of mass loss versus mass tranfer in massive binaries, and the consequences for their binary descendants (WR binaries, massive $\mathrm{X}$-ray binaries, binary pulsars, etc.). In the near future, these bright binaries will be resolved with optical interferometers (such as the GSU CHARA Array, a 7-element instrument with a 400-m baseline), and our current studies will provide the background to help interpret these much anticipated observations.

\section{Acknowledgements}

I am grateful to Mike Wiggs, Bill Bagnuolo, and Laura Penny for their important contributions to this effort. This research was supported by the NASA Astrophysics Data Program grants NAG 5-1218 and NAG 5-1994.

\section{References}

Bagnuolo, W.G., Gies, D.R., Wiggs, M.S. 1992, $A p J$ 385, 708

Chlebowski, T., Garmany, C.D. 1991, ApJ 368, 241

Drechsel, H., Rahe, J., Kondo, Y., McCluskey, G.E. 1981, $A \& A$ 94, 285

Friend, D.B., Castor, J.I. 1982, ApJ 261, 293

Gies, D.R., Wiggs, M.S. 1991, $A p J$ 375, 321

Gies, D.R., Wiggs, M.S., Bagnuolo, W.G. 1993, ApJ 403, 752

Heap, S.R. 1981, in: R.D. Chapman (ed.), The Universe at Ultraviolet Wavelengths: The First Two Years of IUE, NASA CP 2171, p. 485

Howarth, I.D., Stickland, D.J., Prinja, R.K., Koch, R.H., Pfeiffer, R.J. 1991, The Observatory 111, 167

McCluskey, G.E., Kondo, Y. 1976, $A p J$ 208, 760

Sahade, J., Brandi, E. 1991, ApJ 379, 706

Stevens, I.R. 1993, ApJ 404, 281

Stevens, I.R., Blondin, J.M., Pollock, A.M.T. 1992, ApJ 386, 265

Struve, O., Sahade, J., Huang, S.-S. 1958, $A p J$ 127, 148

Struve, O., Sahade, J., Zebergs, V., Lynds, B.T. 1958, PASP 70, 267

Wiggs, M.S., Gies, D.R. 1992, $A p J$ 396, 238

Wiggs, M.S., Gies, D.R. 1993, $A p J$ 407, 252 


\section{DISCUSSION:}

Owocki: I find it really astounding that you don't see much blue-edge variability in these wind binaries. In single stars such blue edge variability is quite common. This has been interpreted as reflecting the relative sensitivity of the wind driving to small changes in conditions, e.g. in density. In binaries, one would expect there would be huge perturbations in the wind, and thus large changes in the driving to terminal velocity.

Gies: In general, my impression is that the O-binaries display discrete absorption components that are similar to those found in the UV wind lines of single O-stars. The occurrence of DACs is usually unrelated to orbital phase. The one exception is $29 \mathrm{CMa}$ where variations in the extreme blue-edge may reflect an orbital variation in the turbulence in the wind.

Kaper: Could you comment on the stationary absorption feature at the blue wing of the CIV profile in the binary system LZ Cep?

Gies: The persistent blue absorption in this feature (at a velocity of $-1800 \mathrm{~km} \mathrm{~s}^{-1}$ ) shows no orbital motion. This lack of variability is common to most O-binaries, and I think it is best explained by scattering ions at great distance from the stars where wind contributions from all orbital phases merge.

Koenigsberger: You are not taking into account the effects of selective atmospheric (wind) eclipses. In WR binaries, this effect is one of the most important (Auer \& Koenigsberger, 1994, ApJ in press) for producing phase-dependent line profile variations in certain lines. Can you comment on this?

Gies: The interpretation of the UV line variations is still rather rudimentary, but basically the observed variations are probably due to atmospheric eclipses. The big differences in my sample of O-binaries compared to the WR + $\mathrm{O}$ binaries is: 1 . winds are of comparable strength, and 2. the wind-strong star is usually the more luminous star in the UV. Thus the largest variations are due to the removal of scattering ions in the wind of the wind-dominant star caused by the wind collision (and bow shock) from the secondary star.

van der Hucht: In your modelling you show profile differences in the $\mathrm{P}$ Cygni absorption part of the composite $\mathrm{O}+\mathrm{O}$ type UV spectra, caused by (i) difference in line optical depth between the two stars and (ii) difference in $v_{\infty}$ between the two stars. In practice, can you separate the two causes?

Gies: The model profiles of Stevens (1993) suggest that a distinction can be made: changes in the blue-edge trace velocity differences while absorption equivalent width variations reflect optical depth differences. However in the four systems studied in depth thus far, we have found only the absorption variations. This is probably a selection effect since in these four systems the stars probably have similar terminal velocities.

Bode: Could you outline briefly the status of the optical interferometer array?

Gies: The CHARA Array has reached an advanced design and engineering phase but construction has not yet begun. Pending some funding decisions, we hope to have the telescope operating by 1997. 